\title{
Application of Photonic Crystal Fiber in Optical Fiber Current Sensors
}

\author{
K. BARCZAK* \\ Department of Optoelectronics, Institute of Physics, Silesian University of Technology \\ B. Krzywoustego 2, 44-100 Gliwice, Poland
}

\begin{abstract}
Optical fibers may be applied in the technique of measuring electric current, particularly as so-called optical current transducers. They are small in size, cheap, light and safe. Their sensitivity, however, connected with the Faraday effect, is rather poor, and they are also susceptible to considerable disturbances (deformations of the fibers). Their sensitivity can be increased by lengthening the path of effect, but this involves also an increase of noise and greater fluctuations of the measured signal due to changes of internal stresses induced by external factors. These negative phenomena can be reduced by applying fibers less sensitive to deformations. The author supposes that photonic crystal fiber with a glass core may display such properties. Orifices in the cladding can to some extent prevent the propagation of deformations from outside the core, and thus decrease the birefringence of the fiber induced by elastooptic effects. The paper presents preliminary measurements of typical photonic crystal fiber exposed to mechanical vibrations.
\end{abstract}

PACS: 42.70.-a, 42.79.Pw, 33.20.Kf, 42.25.Bs

\section{Introduction}

Optical fiber current sensors are constructed basing on a waveguide loop, whose coils coincide with the magnetic field resulting from the electric current flowing through the conductor. The magnetic field causes a twisting of the plane of polarization of the light. This twist reaches its maximum when the direction of the magnetic field coincides with the direction of the ray of light. This condition is satisfied in the waveguide loop mentioned above. What is therefore to be done is to choose the adequate ray of light and the proper number of coils, in order to attain the required sensitivity [1-5]. Unfortunately, however, any increase of the length augments also the noise due to the birefringence of the fiber induced by internal stresses as well as those penetrating from outside. Internal stresses arise in the course of production and are connected with the contrast of the refraction index between the core and the cladding, and due to imperfections of the technological process [5-8]. The induced birefringence is responsible for the change of the state of the polarization of light, causing considerable disturbances in the signal of measurements, which means that it determines the stability of operation of the sensor and the whole measurement system $[8,9]$.

Optical fiber current sensors constitute an alternative of their electric equivalents, viz. optical currents transducers. These latter ones have been applied reliably for many years warranting stable parameters. Indications of these transformers are often utilized in safety devices. Therefore, the stability of operation of optical sensors of electric current is extremely important [10-12].

\section{Photonic crystal fiber specification}

The investigations were carried out by means of commercial photonic crystal fiber (PCF) with a glass core

\footnotetext{
* e-mail: kbarczak@polsl.pl
}

(index guiding), characterized by its single-mode operation at every wavelength, restrained, however by the attenuation of glass [6]. The photonic waveguide is made of silica glass - its spectral attenuation in Fig. 1 is presented. Therefore, the Verdet constant of this material depends to a large extent on the wavelength (visible region), the higher is the Verdet constant, so that PCF permits a free matching of the optimal wavelength [10]. In the visible range of $500 \mathrm{~nm}$ and more the attenuation of the fiber does not exceed several score of $\mathrm{dB} / \mathrm{km}$ (Fig. 1) and can be applied in sensors.

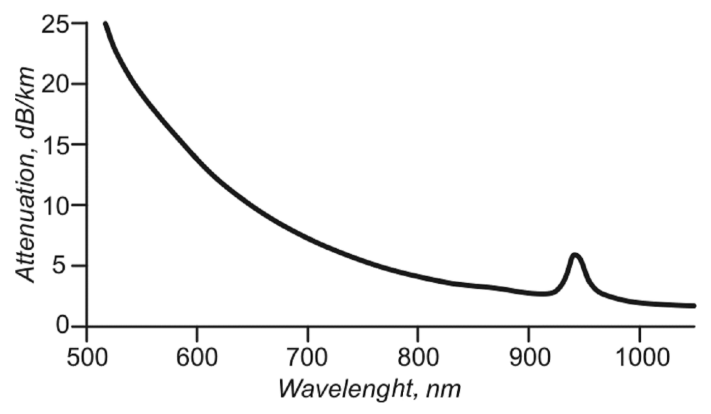

Fig. 1. Attenuation of tested PCF fiber.

Figure 2 illustrates schematically the structure of the waveguide. This is a hexagonal standard structure, the cladding of which has orifices with a diameter of $3.7 \mu \mathrm{m}$ distanced from each other by $8 \mu \mathrm{m}$. Six layers contain 126 orifices.

The author alleges that orifices in the cladding may improve the stability of the state of polarization of the light. The perforated cladding of waveguide can reduce the effect of stresses in the external layer of the fiber on the deformation of the core. Therefore it was attempted to test the fiber in the state of external disturbances in the form of mechanical vibrations. The effect of vibrations and deformations of the fiber is the main problem 
of optical fiber waveguides applied in optical fiber current sensors. It results in a considerable increase of noise and determines the length of the loop in the head of the sensor, and consequently deteriorates greatly the resolution and accuracy of measurements.
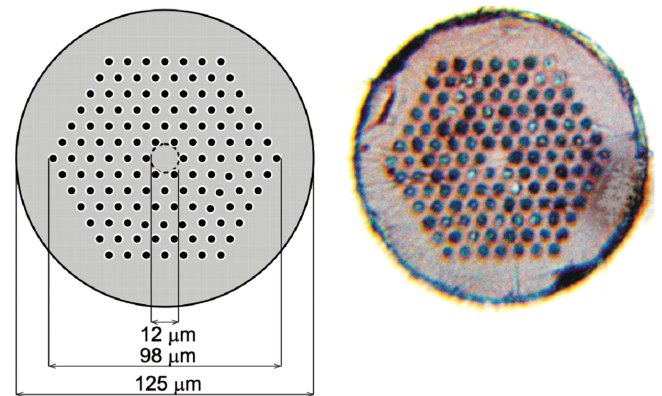

Fig. 2. Cross-section of tested PCF fiber.

\section{Experimental results}

This part of the paper deals with investigations concerning the magnetooptic effect of PCF. Special attention will be paid to the idea of these investigations in the presence of mechanical vibrations of the fiber. The waveguide was tested both in a constant and an alternating external magnetic field. The results of these investigations will be presented.

For the purpose of realising all the intended investigations, an adequate test stand had to be constructed permitting state of polarization (SOP) measurements, the excitation of vibrations and recording the varying signals [8, 11].

This measuring position is presented schematically in Fig. 3. The source of the magnetic field was a long air core coil $80 \mathrm{~cm}$ long, in which a magnetic field is obtained with an induction of up to about $60 \mathrm{mT}$. The measurements were taken at a wavelength of light amounting to $650 \mathrm{~nm}$. The applied waveguide was $2.3 \mathrm{~m}$ long. In all these investigations the tested fiber had to be freely arranged in a straight line in order to minimize the deformation caused by external factors. The investigated photonic crystal fiber was equipped with $\mathrm{FC} / \mathrm{PC}$ connectors, so that at the input a laser could be employed provided with a waveguide terminal consisting of polarization maintaining fiber.

At the output of the waveguide there is the head of analyzer of the state of polarization or the set of polarizer and photodetector. For tests to be carried out in the presence of mechanical vibrations on the section of the tested fiber between the source and the coil the waveguide has been connected to an electromagnetically excited plate (the source of mechanical vibration). This plate vibrates with frequency of about $120 \mathrm{~Hz}$ and amplitudes of about $2 \mathrm{~mm}$. Before and just behind the plates there is a waveguide in two sleeves. Due to such a solution the investigated waveguide vibrated chaotically.

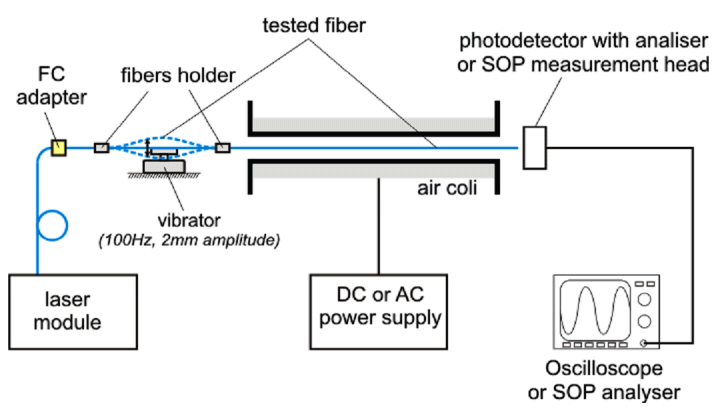

Fig. 3. Measurement stand.

Performed observations indicate that none of the modes of vibration was conspicuously distinctive. The idea of exciting vibrations has been illustrated in Fig. 4.

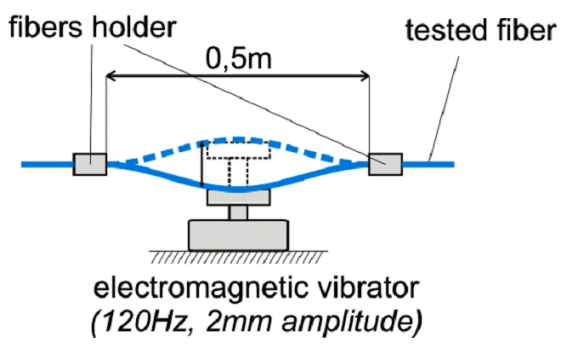

Fig. 4. Electromagnetic vibrator.

The first stage comprised investigations concerning changes of the azimuth SOP without an external magnetic field. The results of this experiment are to be seen in Fig. 5. The arrows denote the intervals of time, in which the vibrator was switched on.

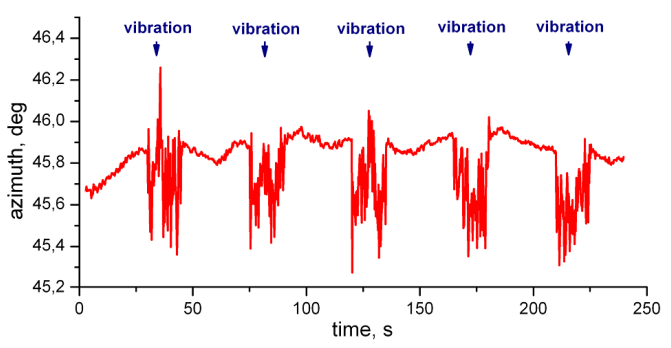

Fig. 5. Azimuth depending on the vibrations.

In the course of the second stage, the magnetooptic effect was tested, first without and then with vibrations. Figures 6 and 7 present the results of these experiments. During the investigations in the presence of vibrations the electromagnetic vibrator was switched on continuously. The determined Verdet constants amounted, respectively, to:

- in the case of measurements performed without vibrations: $V=2.17 \mathrm{rad} /(\mathrm{T} \mathrm{m})$,

- concerning measurements performed in the presence of vibrations: $V=2.23 \mathrm{rad} /(\mathrm{T} \mathrm{m})$. 

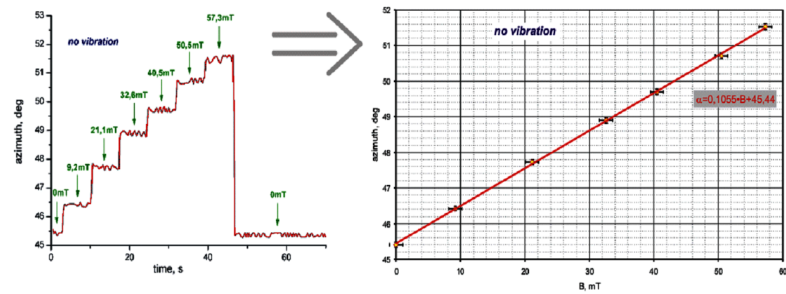

Fig. 6. Magnetooptical effect in the tested fiber (no vibration).

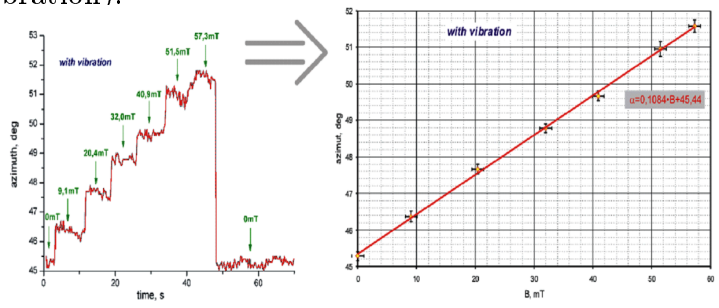

Fig. 7. Magnetooptical effect in the tested fiber (with vibration).

The obtained values of the Verdet constants approximate each other. In Fig. 6 and Fig. 7 it is evident that in the case of measurements with vibrations the values of uncertainty of the azimuth increased, whereas in the case of the absence of vibrations these values were contained within the range from 0.059 to 0.085 degrees. In measurements taken in the presence of vibrations the values of uncertainty of the azimuth amounted from 0.117 to 0.204 degrees. This is twice as high, but the relative value of uncertainty does not exceed $0.5 \%$.

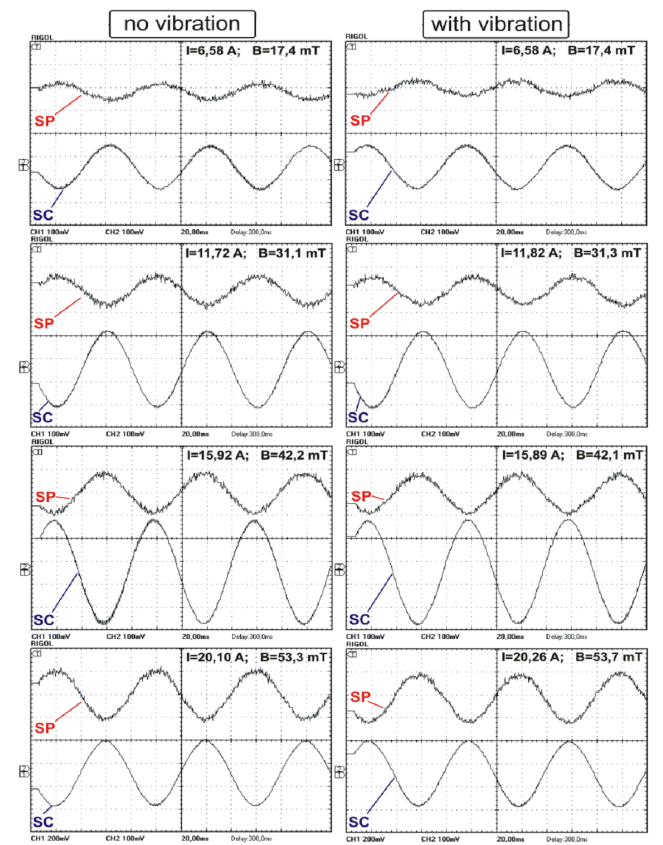

Fig. 8. Signals as a function of time. SC - current signal; SP - voltage signal from photodetector provided at the end fibers. Each diagram concerns other values of the effective current $I$ and also the average value of the effective magnetic field induction $B$.
In the next stage of investigations the tests were performed in an alternating magnetic field. At the output of the measuring position a polarizer and photodetector were installed. The azimuth of the polarizer was adjusted in such a way that at the zero value of magnetic field it would transmit one half of the beam of light. The signals of the photodetector and the signal from the current transducer were passed onto an oscilloscope.

The results of these measurements have been gathered in Fig. 8. The values of the current and the induction of the magnetic field, quoted in each diagram are root-mean-square values. The author consciously left the shift of the phase by 180 degrees, resulting from the direction of the magnetic field and the azimuth of the polarizer. The presented curves prove that the vibrations do not affect the signal recorded on the photodetector.

\section{Conclusions}

The investigations dealt with in this paper have shown that photonic crystal fiber with a glass core may be used in optical fiber current sensors. They display a high sensitivity and weak susceptibility to mechanical vibration. The promising mechanical properties justify the necessity of further investigations (including comparative ones) concerning these waveguides, particularly the possibility of applying them in the construction of optical fiber current sensors. The aim of further investigations is to verify whether PCF fiber ensures more stable responds in the waveguides system of the current sensor than standard fibers consisting of the same material.

\section{References}

[1] K. Barczak, T. Pustelny, J. Phys. (France) IV: JP 137, 15 (2006).

[2] K. Barczak, T. Pustelny, D. Dorosz, J. Dorosz, Acta Phys. Pol. A 116, 247 (2009).

[3] N. Itoh, H. Minemoto, D. Ishiko, S. Ishizuka, in: $O S A$ Technical Digest Series, 12th OFS Conf., Vol. 16, 1997, p. 92.

[4] T. Pustelny, M. Grabka, Acta Phys. Pol. A 116, 385 (2009).

[5] M. Kochanowicz, D. Dorosz, J. Zmojda, J. Dorosz, Acta Phys. Pol. A 118, 1177 (2010).

[6] K. Bohner, T.P. Gabus, H. Brandle, Temperature and Vibration Insensitive Fiber-Optic Current Sensor, ABB Corporate Research, Baden-Dattwil, Switzerland 2000.

[7] T. Pustelny, J. Ignac-Nowicka, B. Jarzabek, A. Burian, Opt. Appl. 34, 551 (2004).

[8] K. Barczak, Bull. Pol. Acad. Sci., Techn. Sci. 59, 401 (2011).

[9] T. Pustelny, I. Zielonka, C. Tyszkiewicz, P. Karasinski, B. Pustelny, Opto-Electron. Rev., 14, 161 (2006).

[10] C.Z. Tan, J. Arndt, J. Phys. Chem. Solids 60, 1689 (1999).

[11] K. Barczak, T. Pustelny, D. Dorosz, J. Dorosz, Acta Phys. Pol. A 114, A-3 (2008).

[12] K. Gut, D. Nabaglo, Acta Phys. Pol. A 116, 307 (2009). 